\title{
I Have Dementia, I Can Help!
}

\author{
Solace Emefa Adzei \\ Department of Graphic Design, University of Education, Winneba, Ghana \\ * E-mail of the corresponding author: sadzei@gmail.com
}

\begin{abstract}
The treatment of dementia has been a matter of concern among health workers, researchers, designers, and art therapists over the world. Though the Ghana Statistical service does not acknowledge dementia as a disability, the disease cannot be swept under the carpet pretending it does not exist. Thus, the aim of this paper is to adapt design interventions involving persons living with dementia to help break the cycle of shame, opinions, attitudes, and ways-of-doing, in involving people living with dementia in Ghana. The paper also proposes a dimension on using visual arts in the form of art therapy as an approach with relevant evidence as an intervention in other countries. Additionally, the paper deliberates on the need for collaborations between government, NGOs and stakeholders for the development of appropriate infrastructure as well as the implementation of policies and art interventions to support persons living with dementia.
\end{abstract}

Keywords: dementia, art therapy, art interventions, policies

DOI: $10.7176 / \mathrm{ADS} / 98-01$

Publication date: January $31^{\text {st }} 2022$

\section{Introduction}

European countries including the United Kingdom spend billions in monetary value managing and trying to find a cure for dementia (Hurd, Martorell, Delavande, Mullen, \& Langa, 2013) and (Schneider, 2018). Dementia is a collective description of various symptoms of cognitive decline affecting memory, thinking and social abilities which can severely interfere with daily functioning of an individual with such a condition (Harwood \& McCulloch, 2017). The disease is usually chronic or progressive in nature, with each case unique on its own and should not be associated with old age as was previously the case, though the chances of developing the disease increases with age. It is estimated that 50 million people are living with dementia with almost 9.9 million people developing dementia each year, which implies there is a new case of dementia every four (4) seconds somewhere in the world (World Health Organization, 2015).

There has been several research in European countries that aim to improve the quality of life of people living with dementia; however, the advancement of these researches face challenges such as the delivery of appropriate care contributing to unmet needs of people living with dementia; inadequate infrastructural needs due to the lack of local service providers; inadequate skillsets in hospitals needed in dementia care and lastly, insufficient evidence-based strategies to help cater for the increasing number of dementia cases to advance dementia care which will maximize independent living (Martin, O’Connor, \& Jackson, 2020). Research also indicates the reluctance of healthcare practitioners who primarily care for people living with dementia to share information due to the lack of confidence and the perception that the person will die soon, thus, they avoid giving any information concerning their patient; besides, there is also a common disregard of the needs, values and wants of people living with dementia, especially during the last days of the person's life (Martin, O'Connor, \& Jackson, 2020).

Dementia Association of Ghana postulates an estimated one (1) in nine (9) persons over sixty-five (65) years and one (1) in four (4) over eighty years, develop this progressive illness with no cure (Ghana News Agency, 2014). However, the Ghana Statistical Service (2014) does not have any documentation or policy on the classification of dementia as a disability though persons living with dementia are challenged in several aspects of their lives starting with a decline in their cognitive abilities. This is because dementia in Ghana, and other developing countries are taken and treated lightly with symptoms such as being forgetful in the elderly taken as the sheer effect of old age (World Health Organization, 2015). The Ghanaian method of caring for the elderly has been based on the traditional approach, where care for the elderly is by extended family members. This includes but not limited to provision of physical, emotional and financial support. The extended family system in recent times, has seen a steady degeneration due to urbanization and the gradual waning of the traditional system through western education and the peaking rate of Christianity. This has resulted in older family members barely having support from their younger family members (Akinny, 2016), with no major infrastructure in place to cater for them, let alone people with dementia though some NGOs have taken it upon themselves to help (Spittel \& Wolf-Ostermann, 2013).

Since dementia involves the cognitive decline of a person over time, certain interventions can be made depending on the severity of the case. Interventions such as art; whether visual or performing may be used to entertain, comfort, stimulate, soothe or support a person with dementia (Brooker \& Latham, 2015). Such 
interventions including art therapy may be facilitated by a professional artist who is not just an artist but also has some experience and is familiar with persons living with dementia. Art therapy is suggested as an intervention because of its focus on creative process for healing used to improve cognitive and sensory-motor functions, foster self-esteem and self-awareness (America Association of Art Therapy, 2017). Essentially, art therapy helps to promote emotional resilience, promote insight, improve social skills, reduce and resolve conflicts and distress, and advance societal and ecological change (America Association of Art Therapy, 2017).

Art has been used to tell stories, seek out information and to give answers to questions ( Arts Education Partnership, 2004). The underlining factor is that art has been used to communicate through the years (Shaw, 2003) and has been established to improve the mental, emotional and spiritual health of patients. Art as therapy is suggested as an intervention because creativity (Basting, 2009) is not dependent on memory which proves that creative processes are used to induce memories to allow people to share their experience, to reinforce identity, and to strengthen relationships with their loved ones, and also with care givers and others involved in the intervention process (Schneider, 2018).

In Ghana, art and design are interchangeably used (Ross, 2004) and forms part of the Ghanaian's way of life, serving as proof of their beliefs, aspirations and needs; physically, emotionally, and psychologically (Ayiku, 2008). Ghanaians use both visual and communicative symbols to portray their philosophical thinking and way of life which are used as motifs in textile products, wood carvings, and ceramics among other forms of art. An example is the Adinkra symbol which is a typical representation of ideas and communication in abstract shapes and form. Obviously, culture and art are inseparable; art forms the core of the culture and made to serve specific purposes. For example, the Akuaba doll was given to a barren woman to make her fertile. The name "Akuaba" comes from the Akan legend of a barren woman named Akua; desirous of bearing children consulted a priest and was instructed to commission the carving of a small wooden child which was to be carried on her back as if it were a living child. Akua cared for the figure as she would a living baby, even giving it gifts of beads and trinkets. Eventually, Akua conceived and gave birth to a beautiful baby girl. Soon thereafter, other barren women began adopting the same practice to overcome barrenness.

Though, the intervention through art may not cure the disease, it is important to consider, just like the Akuaba doll, to involve persons living with dementia in the design process to help them manage the numerous changes that impugn their day-to-day activities. Thus, the aim of this report is to encourage visual arts through art therapy in the form of design interventions involving persons living with dementia to help break the cycle of shame, opinions, attitudes, and ways-of-doing in Ghana.

\section{Why involve persons with dementia in the design process?}

Designs are essentially meant to solve problems through creativity and at the same time promote economic growth by generating a number of possible solutions which exploit various techniques or mechanisms that encourage the creation of innovative solutions (Ambrose \& Harris, 2010). Visual concepts which involve the organization and clear presentation of content is one appropriate way to involve persons living with dementia in the design process; to maximize their understanding by essentially providing the visual communication necessary to transmit messages and ideas in an attractive and acceptable way (Kirk \& Kennedy, 2001).

Various studies reveal an encouraging relationship between participation in the visual arts (painting, colouring, and sculpting, and other art forms) in the form of art therapy and improvements in the well-being, cognitive function and communication (Basting, 2009) of persons living with dementia. Art-based activities that promote memory skills and social abilities have gained a prominent role in dementia research lately and as a result provided a non-verbal channel of communication with improvement in attention, quality of life and social skill; though, there is more room for further study to strengthen existing evidence (Shoesmith, Charura, \& Surr, 2021). These art-based activities in the form of visual art interventions are complex in nature due to the several independent and interacting components. This is due to the limited visual art intervention research in dementia, leading to the lack of understanding of the processes and elements that lead to beneficial outcomes as problematic and attribute this to methodological weakness and the relatively newness of field (Zeilig, Killick, \& Fox, 2014). However, understanding the variations and parameters in facilitator skill set, content of the creative activities, participant demographics and the setting in which the intervention is delivered will help in the successful implementation which will invariably influence the intervention outcomes (Shoesmith, Charura, \& Surr, 2021).

Various initiatives including laws and policies that support persons living with dementia have been effectively implemented in North European countries (Riquelme-Galindo \& Lillo-Crespo, 2021) leading to the design and development of dementia-friendly environment interventions that puts the needs of persons with dementia first to facilitate their daily routines (Riquelme-Galindo \& Lillo-Crespo, 2021). Thus, a well-designed environment may help with memories and physical abilities, improve quality of life, provide a therapeutic atmosphere which will enhance their wellbeing (Wahl, 2015). This will require understanding the behaviours and affective responses among persons living with dementia taking into consideration varying individual 
competence, based on multiple factors including cognitive status, type of diagnosis, functioning abilities and challenges, personal preferences, and life history (Chaudhury \& Cooke, 2014). Apart from affecting the internal aspect of their lives, their external environment is also greatly affected and plays a major role in how they are able to balance these two factors. There is therefore the need for comfort and mastery of one's residential environment with a relative adaptive coping strategy to sustain or attain residential normalcy in existing neutral physical environment (Golant, 2015)

Consequently, involving persons with dementia in the design process can help achieve some residential normalcy if they feel comfortable and in control of their environment and may not have the need to change anything (Brandtstädter \& Greve, 1994). Most importantly, involving persons living with dementia in the design process helps create a sense of purpose, builds their confidence, empowers and increase their self-esteem which improves health and wellbeing and enables these people to 'give back' to leave a legacy. Essentially, this will also eradicate the fear associated with persons living with dementia to give some education to eliminate stigmatization and stereotyping (Batsch \& Mittelman, 2012). Further, people with dementia have the fear they will not be taken seriously, therefore shy away from people who may listen and help build relationships (Milton, 2016).

Studies suggest that stories of the lives of persons living with dementia help people who interact with them to see them as individuals, and gives them a platform to be heard and recognised as people with unique stories (Gridley, Brooks, Birks, Baxter, \& Parker, 2016). Additionally advocates of social health are of the view that a decline in functioning abilities does not instantly change the quality of life. Essentially, supporting persons living with dementia to develop strategies helps them to maintain a balance between opportunities and limitations to manage life with some independence to participate in social activities for individual growth (Brooker \& Latham, 2015). Thus, their skills are recognised and their unique expertise and experiences from the 'lived experience' are shared through various roles, which occupies them and gives them a sense of value and purpose (Milton, 2016).

\section{The design process with persons with dementia}

Dementia includes a range of brain diseases that brings about changes in behaviour and functional limitations. These deteriorating conditions occur over an unspecified period of time where the person loses their identity, interpretative skill, abilities and independence in very different ways (Rodgers, 2021). Common symptoms of dementia can include short term memory loss which makes information difficult to retain and restricts language which may cause confusion. Also, familiar environments may become unfamiliar causing people with dementia to get lost. They may also lose interest in social engagements, become quieter and more introverted (Batsch \& Mittelman, 2012). This is where art can be used as an intervention, though their needs may require varied support depending on the stage of diagnosed conditions. The approach to providing support by involving them in the design process has important implications that can impact the basis of their daily activities.

To begin an intervention, certain parameters must be put in place to ensure a fruitful outcome which is dependent on the level or stage of diagnoses of the disease (Schneider, 2018). Schneider (2018) suggests the consideration of three contextual factors: the level at which the intervention is delivered, the severity of dementia in the person participating, and who is delivering the activity. He also stressed on the need to be mindful of the relevant place and space, differences in the intentions and outcomes of the activity, as well as the involvement of close persons and care partners.

\subsection{Collecting stories}

To involve and engage persons living with dementia in the design process will require effective communication which is an important key to the success of a design project. Literature indicates that communicating with persons living with dementia is a skill fundamentally lacking among anxious healthcare staff who lack the knowledge of how to establish meaningful communication and sharing of relevant information (Martin, O'Connor, \& Jackson, 2020). Thus, facilitators as well as designers and not just people with good communication skills, who care about people with a good set of eyes and ears will do a great job in engaging with persons living with dementia; the better the communication skills the more effective it will be to get to know the people working together and the easier it will be to engage them.

Involving persons living with dementia in the design process also presents the opportunity to nurture the hidden capabilities of participants to support the realization of skills and outcomes that stimulate esteem and self-actualisation. To achieve this, artistic participation is suggested to create a temporary feeling of togetherness between the artists, facilitators and persons living with dementia. Differences in personality and differing views are left behind during activity time (Klaveren, 2012) to help generate dialogue and encourage social activism.

Artistic participation can take several forms, such as focus group discussion, one-on-one conversations, observation etc. through which information can be collected to help in the co-designing projects. Using focus groups for discussions with persons with living dementia can actually help them to clearly express their point of 
view without the pressure of having to fit into a stereotype. They are also happy to work with people who are 'just like them' which boost their confidence, helps them articulate their views and maintain focus (Shoesmith, Charura, \& Surr, 2021) in a dementia friendly meeting space that helps in facilitating the purpose of the discussion with the necessary support.

On the other hand, one-on- one conversations with a person living with dementia is suggested to facilitate the communication needs of a person in the advanced stage of the disease who may find it challenging to work in a group; consequently, ensures the person is not side-lined by the vociferous members in the group (Rodgers, 2012). This also gives the person involved space to think, construct responses, build and establish good communication with facilitator(s) at a pace appropriate for both parties involved especially for the person with dementia (Rodgers, 2012).

\subsection{Translating stories into opportunities and solutions}

Projects involving persons living with dementia should cover a number of processes and investigations into how designs can be used to unlock and divulge personal and collective capabilities, utilising and motivating interests and unveiling new ways of doing things. This approach importantly promotes the idea that design is a social activity and provides a space for shared enjoyment and fun in safe experimental conditions (Rodgers \& Tennant, 2014). However, there is the tendency for one person (the leader) to take the dominant role during the execution process which can be prevented with clearly set aims and objectives. Most importantly, the rationale of the project should be known and agreed upon by both parties involved for an ethical and transparent relationship beneficial for all (Klaveren, 2012). This puts a value on each person's input where there is no failure but a learning process for all involved. This may result in a number of thought-provoking outcomes (creations) that challenge assumptions concerning the needs of persons living with dementia and also encourage a sense of self and value through personal experiences. An example of such intervention that translates the experiences of people living with dementia into art is the Disrupting Dementia tartan design project. The main aim of this project was to change astute attitudes and perceptions about dementia by bringing to prominence the creative abilities of people living with dementia. The project stressed on the need for careful and well-designed plans, preparation, thus encouraged people living with dementia to contribute their quota of social responsibility to the United Kingdom (UK) after diagnosis. Their products through this project have been sold with a contribution of $£ 461$ million value of exported design products and services from the UK to various parts of the world (Milton, 2016).

Such projects can be replicated in Ghana spearheaded by organizations such the Ghana dementia Association in collaboration with companies such as Nestle Ghana, Coca Cola Ghana to commission persons living with dementia to come up with commemorative designs that share their life experiences expressed through designs celebrating their abilities with some value on their contribution to society.

\section{Testing}

The ensuing designs and prototypes will be evaluated in real-life context to ascertain their usefulness and improve the viability of designs (Koskinen, Zimmerman, Binder, Redstrom, \& Wensveen, 2013). This includes methods that monitor and evaluate viability of designs to improve co-designed works through reflections of daily written inputs and questions about conceptualization through to final designs that give the reasons behind their creations and how they integrate into their daily activities.

The evaluation tools include but not limited to monitoring case studies to highlight participants' stories that impact the codesigned projects in which they are involved. This is to help provide carefully selected, powerful testimony as well as rich descriptions of activities, processes and experiences (Public Health England, 2016).

Also, creative and arts-based methods using techniques such as photography, film, visual arts, poetry, creative writing, music, drama and dance can be used to support evaluation since these codesigned projects are intended to produce artworks and artefacts that inform understanding of project impacts (Leavy, 2017). Effectively, these can help uncover hidden perspectives, by adding empathic power to strengthen participants' voices. Essentially, these will be used in the dissemination of evaluations for easy accessibility of information to audiences beyond policy making circles.

Lastly, an economic assessment can help capture benefits and savings from using arts-based approaches within codesigned projects. This is to help in the projection of social return on investment (SROI) on costs and impact of an intervention whether it takes place or not (Goodacre \& Mccabe, 2002).

For the successful implementation of art and codesigned interventions, various stages of assessment are important to determine the success of the project, correct mistakes and make improvements that will impact future projects. The cycle of assessment suggested include the formative evaluation stage that involves monitoring and audit through interviews, use of questionnaire and discussions to measure how projects will do in relation to target projection (Boothroyd, 2018). This is to ensure that activities designed for the art intervention programme are feasible, appropriate, and acceptable before they are fully implemented (Public Health England, 
2016). This stage can also be used to help capture participants' experiences during art intervention projects to help modify an existing programme for an enhanced experience for future development.

Further, evaluations can be undertaken during the implementation of the intervention to determine whether programme activities have been implemented as intended and resulted in certain outputs (Public Health England, 2016). Process evaluations may be periodically conducted throughout the programme by reviewing the activities and output components. In addition, a broader impact of the project on facilitators as well as stakeholders and not just participants may be explored while participants are at the centre of the process (Family health international, 2004). This allows understanding of impacts of art interventions and codesigned projects to develop through dialogue and not in response to themes and results that are pre-determined by assessors and sponsors.

Lastly, an assessment at the end of the project will help establish whether the project met its aims and objectives as well as its impact on participants. Thus, an outcome evaluation is required quantitatively to produce evidence of a successful implementation of the programme which can be carried out with individual participants or in groups (Boothroyd, 2018).

In view of this, organizations such as Talking Mat, Deep and Disrupting Dementia (Rodgers, 2016) have found collaborative ways to involve persons living with dementia to engage in activities that promote a sense of inclusion, value of opinions and empowerment. In the case of Disrupting Dementia tartan co-design (Rodgers, 2016), workshops began with a short presentation of the project aims and requirements associated with the creation of the project. The project was created because it fit into the Scottish culture, thus giving participants a form of identity with personal attachments and stories of their own. There were some evaluations to give the needed support (physical and emotional) for each task with appropriate use of language. Participants were free to use any colours and shapes of their choice during the design stage which began with physical models made using coloured ribbons followed by the digital versions using free accessible Internet-based tartan design tool (Rodgers, 2012). Like Disrupting Dementia, Talking Mat and Deep, UK, similar collaborative workshops can be replicated in the various communities in Ghana, for persons living with dementia to co-design and produce items with sentimental value associated with Ghanaian traditions and values.

\section{The results}

These interventions may generate a range of positive comments from co-designers (persons living with dementia), carers, and family members throughout the process of designing which will engender positive feelings towards the design activities and put value on the design process. Consequently, each participant is afforded the opportunity to make a decision and contribute to the design leading to a significant outcome within a project. Design sessions are also enjoyable and offer a safe space for various ideas to be expressed where differing opinions are tolerated. Besides, feedback during sessions will allow the collection of diverse sets of opinions, observations, and comments in terms of perceptions about people living with dementia and their place in the larger society, thus divulge new creative and innovative ways to help them to take control of their own design output, and lay claim to ownership of the creative outcomes produced. A sense of independence in thinking and acting through the choice of personal preferences will also be demonstrated through the creation of their designs, with a sense of eagerness and enjoyment as well as pride and esteem in co-designed work. Additionally, found objects including materials produced locally can be used during these projects to encourage the production and use of locally manufactured materials which will also contribute to the economic growth of Ghana.

\section{Conclusion}

People with dementia have interests and needs that should not be overlooked. Successful network of persons living with dementia can be nurtured to encourage them to speak and share their experiences as is the case in countries like the United Kingdom, America and China. Though the Ghana Statistical Service does not have any documentation or policy on the classification of dementia as a disability, it is not too late taking a look at this disease and putting policies in place that will address various challenges of persons living with dementia through interventions. These interventions through art therapy in the form of design projects for people living with dementia will address issues of inclusion, participation, and creativity that will promote a sense of self identity through activities that are carefully designed, developed and facilitated in dementia-friendly settings.

These projects will act as catalysts for conversations, personal interest and recollection on past working lives, which when exploited will provide amazing benefits for persons who are living with dementia. People living with dementia could be the "vernacular designers" who are free to express themselves without the constraints of discipline to help conduct co-designed projects that are truly meaningful through their shared experiences, full participation and inclusion. This may result in design solutions that will exhibit the creative abilities of persons living with dementia as well as the rewarding ways in which they can co-operate with and inform the world. Thus, offering persons living with dementia a better quality of life. Consequently, these co- 
designed activities and contributions will reinforce their right to active participation and social responsibility, through the inclusive nature of the co-designed activities to provide meaningful and appropriate engagement for each of them to help preserve their sanity and connection to life and society as long as possible.

\section{Recommendation}

Political goals shape the focus in priorities given to the health sector of a country, in this case dementia. Essentially, political and interest groups should consider dementia as a disability and make provision for medical care with integrated social care to establish a distinct benchmark that address challenges in dementia care in Ghana. This way, appropriate policies will be formulated to take care of the needs of persons living with dementia in Ghana.

This will in turn create awareness about the disease and also urge its acceptance as a form of disability by the government of Ghana through collaborations with the national Alzheimer association to help implement regional or national campaigns to reduce stigma and raise awareness. Thus, the reduction in stigmatization of the disease will encourage persons who have the disease to come out for diagnosis and also share their experiences for inclusion in the performance of their social responsibilities. Consequently, this will help erase misconceptions associated with persons living with dementia thereby encouraging assistance, care and love from communities instead of confining them to witch or isolation camps.

Also, government in collaboration with non-governmental organisations, must ensure projects are developed with all available resources with timely distribution for the intended audience.

Additionally, the right infrastructure taking into consideration the physical environment of long-term care facilities will play an important role in affecting positively the quality of life of persons living with dementia and the quality-of-care practices. This is because the environment influences by serving as a therapeutic source that reduces behavioural and affective symptoms associated with cognitive decline and loss since persons living with dementia are very sensitive and easily agitated by their environment; it is therefore important to ensure the designed environment is suitable and responsive to cognitive needs and functioning.

Apart from infrastructure, these projects when properly implemented will create a niche for visual artist who will serve as facilitators, thus creating a source of employment. The universities will churn out the needed human resource to fill this gap thereby reducing unemployment levels in the country leading to economic growth.

\section{References}

Arts Education Partnership, 2004. The Arts and Education: New Opportunities for Research, Washington, DC 20001-1431: One Massachusetts Avenue, NW, Suite 700.

Akinny, W. D., 2016. Investigating the desirability and feasibility of the 'Old People's Home' as a viable business in Ghana, Berekuso: Ashesi University College.

Ambrose, G. \& Harris, P., 2010. Design Thinking. Case Postale, Switzerland: AVA Publishing SA.

America Association of Art Therapy, 2017. https://www.arttherapy.org/upload/2017_DefinitionofProfession.pdf. [Online] Available at: https://arttherapy.org/about-art-therapy/

Basting, A., 2009. Forget Memory: Creating Better Lives for People with Dementia.. Washington, DC, USA: Johns Hopkins University Press.

Batsch, N. L. \& Mittelman, M. S., 2012. World Alzheimer Report 2012: Overcoming the stigma of dementia, London: Alzheimer's Disease International.

Boothroyd, R. A., 2018. Process and Outcome Evaluation Approaches. South Florida: Louis de la Parte Florida Mental Health Institute, University of South Florida.

Brandtstädter, J. \& Greve, W., 1994. The Aging Self: Stabilizing and Protective Processes. Developmental Review, pp. 52-80.

Brooker, D. \& Latham, I., 2015. Person-centred dementia care: Making services better with the VIPS framework. London, England: Jessica Kingsley.

Chaudhury, H. \& Cooke, H., 2014. Design matters in dementia care: The role of the physical environment in dementia care. Excellence in dementia care (2nd Edition), pp. 144-158 UK: Open University Press..

Collective Encounters, 2013. AD-Report. [Online] Available at: http://collective-encounters.org.uk/wpcontent/uploads/2014/02/AD-Report.pdf

Family health international, 2004. Module 10: Monitoring and Evaluating Clinical Care Programs. s.1.:USAID Implementing AIDS Prevention and Care (IMPACT) Project.

Ghana News Agency, 2014. Over 17,000 people with dementia in Ghana. [Online] Available at: http://www.ghananewsagency.org/health/over-17-000-people-with-dementia-

Golant, S. M., 2015. Special Issue: Successful Aging; Residential Normalcy and the Enriched Coping Repertoires of Successfully Aging Older Adults. The Gerontologist Society of America, p. 70-82.

Goodacre, S. \& Mccabe, C., 2002. An introduction to economic evaluation. Emergency medicine journal, pp. 
EMJ. 19. 198-201. 10.1136/emj.19.3.198..

Gorb, P. \& Dumas, A., 1987. Silent Design. Design Study, 8,, p. 150-156.

Gridley, K. et al., 2016. Improving care for people with dementia: development and initial feasibility study for evaluation of life story work in dementia care. Health Services and Delivery Research, Volume 4, Issue 23, p. 1.

Guerchet, M. et al., 2017. Dementia in sub-Saharan Africa, Challenges and Opportunities. Alzheimer's Disease International (ADI), p. 69.

Harwood, D. \& McCulloch, Y., 2017. Factsheet400LP. [Online] Available at: https://www.alzheimers.org.uk/sites/default/files/pdf/what is dementia.pdf

Hurd, M. D. et al., 2013. Monetary costs of dementia in the United States. The New England journal of medicine, 368(14), p. 1326-1334.

Kirk, J. \& Kennedy, G., 2001. ADDING VALUE TO EDUCATIONAL MULTIMEDIA: THE ROLE OF GRAPHIC DESIGN, Melbourne: ASCILITE Conference proceedings.

Klaveren, R. $\quad$ v., 2012. Research Gate. [Online] Available at: https://www.researchgate.net/publication/254464448

Koskinen, I. et al., 2013. Design Research Through Practice: From the Lab, Field, and Showroom. Professional Communication, IEEE Transactions on 56(3),, pp. 262-263.

Leavy, P., 2017. Introduction to Arts-Based Research. In: Handbook of Arts-Based Research. New York: Guilford Publications, pp. 1-19.

Martin, A., O'Connor, S. \& Jackson, C., 2020. A scoping review of gaps and priorities in dementia care in Europe. Dementia, pp. 2137-2148.

Milton, S., 2016. Inclusion of People with Dementia in the Design of Services, Dublin 2, Ireland: Genio, Unit 1921,Westland Square, Pearse St.

Milton, S., 2016. Innovations in Dementia CIC. [Online] Available at: https://www.genio.ie/system/files/publications/GENIO_DEMENTIA_INCLUSION_SERVICE_DESIGN.p df

Public Health England, 2016. Arts for health and wellbeing; An evaluation framework, London: PHE publications gateway number: 2015595 .

Riquelme-Galindo, J. \& Lillo-Crespo, M., 2021. Designing Dementia Care Pathways to Transform Non Dementia-Friendly Hospitals: Scoping Review. International Journal of Environmental Research and Public Health, pp. 2-5.

Rodgers, P., 2012. Designing Work with People Living with Dementia: Reflecting on a Decade of Research.. International Journal of Environmental Research and Public Health, pp. 1-16.

Rodgers, P. A., 2016. "Disrupting Dementia" Tartan Co-Design Exhibition, Lancaster: Smith Art Gallery and Museum, Stirling.

Rodgers, P. \& Tennant, A., 2014. Bogota, Columbia, s.n.

Ross, M., 2004. Art at the Crossroads: The Contested Position of Indigenous Arts in Ghana's Post-Colonial Education Systems. Journal for Studies in Art Education, pp. 117-134.

Schneider, J., 2018. The Arts as a Medium for Care and Self-Care in Dementia: Arguments and Evidence. International Journal of Environmental Research and Public Health, pp. 1-11.

Schneider, J., 2018. The Arts as a Medium for Care and Self-Care in Dementia: Arguments and Evidence. International Journal of Environmental Research and Public Health, pp. 1-11.

Shaw, P., 2003. What's art got to do with it. [Online] Available at: http://www.creativenz.govt.nz/assets/ckeditor/attachments/1061/the_role_of_arts_in_neighbourhood_renew al_by_shaw.pdf? 1411696269

Shoesmith, E. K., Charura, D. \& Surr, C., 2021. What are the elements needed to create an effective visual art intervention for people with dementia? A qualitative. Dementia, p. 1336-1355.

Spittel, S., 2014. "I an not a witch" Stigmatization of people with dementia in Ghana. [Online] Available at: https://www.alz.co.uk/sites/default/files/conf2014/OC132.pdf

Spittel, S. \& Wolf-Ostermann, K., 2013. Challenges in health care for people with dementia in Ghana Alzheimer's \& Dementia. The Journal of the Alzheimer's Association, March.p. 488.

Spittel, S. \& Wolf-Ostermann, K., 2013. Challenges in health care for people with dementia in Ghana Alzheimer's \& Dementia. The Journal of the Alzheimer's Association, March.p. 488.

Wahl, H.-W., 2015. Theories of Environmental Influences on Aging and Behavior. Encyclopedia of Geropsychology, pp. 1-8.

World Health Organization, 2015. Alzheimer's Disease International. [Online] Available at: http://www.who.int/mental_health/neurology/dementia/dementia_thematicbrief_executivesummary.pdf

Zeilig, H., Killick, J. \& Fox, C., 2014. The participative arts for people living with a dementia: A critical review. International Journal of Ageing and Later Life, 9(1), pp. 7-34. 


\section{Author}

Solace Emefa Adzei is a lecturer at the Department of Graphic Design - University of Education, Winneba, Ghana. She holds two master degrees from the Oxford Brookes University, Oxford - UK in Digital Media Production (2012) and the other from Kwame Nkrumah University of Science and Technology, Kumasi - Ghana in Publishing (2017). She majored in graphic design and animation. She teaches animation at the Department of Graphic Design - University of Education, Winneba - Ghana. 